\title{
Silencing of Vangl2 attenuates the inflammation promoted by Wnt5a via MAPK and NF-KB pathway in chondrocytes
}

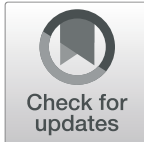

Ke Zhang ${ }^{1 \dagger}$, Zhuoying Li ${ }^{1 \dagger}$, Yunyang Lu', Linyi Xiang ${ }^{2}$, Jiadong Sun ${ }^{1}$ and Hong Zhang ${ }^{*}$

\begin{abstract}
Background: The Wnt planar cell polarity (PCP) pathway is implicated in osteoarthritis (OA) both in animals and in humans. Van Gogh-like 2 (Vangl2) is a key PCP protein that is required for the orientation and alignment of chondrocytes in the growth plate. However, its functional roles in OA still remain undefined. Here, we explored the effects of Vangl2 on OA chondrocyte in vitro and further elucidated the molecular mechanism of silencing Vangl2 in Wnt5a-overexpressing OA chondrocytes.
\end{abstract}

Methods: Chondrocytes were treated with $\mathrm{IL}-1 \beta(10 \mathrm{ng} / \mathrm{mL})$ to simulate the inflammatory microenvironment of OA. The expression levels of Vangl2, Wnt5a, MMPs, and related proinflammatory cytokines were measured by RT-qPCR. Small interfering RNA (siRNA) of Vangl2 and the plasmid targeting Wnt5a were constructed and transfected into ATDC5 cells. Then, the functional roles of silencing Vangl2 in the OA chondrocytes were investigated by Western blotting, RT-qPCR, and immunocytochemistry (ICC). Transfected OA chondrocytes were subjected to Western blotting to analyze the relationship between Vangl2 and related signaling pathways.

Results: $I L-1 \beta$ induced the production of Vangl2, Wnt5a, and MMPs in a time-dependent manner and the significantly increased expression of Vangl2. Vangl2 silencing effectively suppressed the expression of MMP3, MMP9, MMP13, and IL-6 at both gene and protein levels and upregulated the expression of type II collagen and aggrecan. Moreover, knockdown of Vangl2 inhibited the phosphorylation of MAPK signaling molecules (P38, ERK, and JNK) and P65 in Wnt5a-overexpressing OA chondrocytes.

Conclusions: For the first time, we demonstrate that Vangl2 is involved in the OA process. Vangl2 silencing can notably alleviate OA progression in vitro by inhibiting the expression of MMPs and increasing the formation of the cartilage matrix and can inhibit the proinflammatory effects of Wnt5a via MAPK and NF-KB pathway. This study provides new insight into the mechanism of cartilage inflammation.

Keywords: Chondrocytes, Osteoarthritis, Vangl2, Collagen, MMPs, Wnt5a/PCP

\footnotetext{
* Correspondence: zhangh27@mail.sysu.edu.cn; zhangk236@mail2.sysu.edu.cn

${ }^{\dagger} \mathrm{Ke}$ Zhang is the first author and Zhuoying Li is the co-first author.

'Hospital of Stomatology, Sun Yat-sen University, Guangdong Provincial Key

Laboratory of Stomatology, Guanghua School of Stomatology, Sun Yat-sen

University, No.56 Lingyuan West Road, Guangzhou 510055 Guangdong,

People's Republic of China

Full list of author information is available at the end of the article
}

(c) The Author(s). 2021 Open Access This article is licensed under a Creative Commons Attribution 4.0 International License, which permits use, sharing, adaptation, distribution and reproduction in any medium or format, as long as you give appropriate credit to the original author(s) and the source, provide a link to the Creative Commons licence, and indicate if changes were made. The images or other third party material in this article are included in the article's Creative Commons licence, unless indicated otherwise in a credit line to the material. If material is not included in the article's Creative Commons licence and your intended use is not permitted by statutory regulation or exceeds the permitted use, you will need to obtain permission directly from the copyright holder. To view a copy of this licence, visit http://creativecommons.org/licenses/by/4.0/ The Creative Commons Public Domain Dedication waiver (http://creativecommons.org/publicdomain/zero/1.0/) applies to the data made available in this article, unless otherwise stated in a credit line to the data. 


\section{Introduction}

Osteoarthritis (OA) is the most prevalent joint disorder characterized by chronic inflammation, progressive destruction of articular cartilage, and subchondral bone sclerosis [1, 2]. The complex pathogenesis of OA involves mechanical, inflammatory, and metabolic factors, which ultimately lead to structural destruction of articular cartilage and failure of the synovial joint [3]. Chondrocytes are the only resident cells in the articular system and are critical for maintaining the dynamic equilibrium between anabolism and catabolism in the extracellular matrix (ECM) [2, 4]. Several risk factors such as abnormal mechanical stress and proinflammatory cytokines have been shown to reduce chondrocytes and degrade the ECM in cartilage $[5,6]$. Although increasing efforts have been dedicated to revealing the pathological process of irreversible structural change, the molecular mechanisms underlying this disease still remain elusive.

$\mathrm{OA}$ is an active dynamic alteration, which arises from an imbalance between the repair and destruction of joint tissue, regulated by a complex network of different molecular pathways, including the Wnt signaling network [7, 8]. Wnt5a, a representative of the Wnt family, activates the non-canonical Wnt pathway which includes the planar cell polarity (PCP) pathway and the $\mathrm{Ca} 2+$ pathway. Wnt5a has been shown to play a critical biological role in cartilage inflammation as it promotes the catabolic signaling of chondrocytes [9]. Increased Wnt5a signaling in OA has been implicated in the upregulation of inflammatory cytokines, chemokines, and matrix metalloproteinases (MMPs) as well as reduction of type II collagen (Col-2) and aggrecan [9-11].

Van Gogh-like 2 (Vangl2) is a core PCP component that regulates Wnt/PCP signaling [12-15]. Recently, evidence has accumulated that the pathogenesis of $\mathrm{OA}$ is associated with abnormal activation of Wnt/PCP pathway, which regulates the expression of MMPs and cartilage-related genes $[9,10,16-18]$. Notably, previous research suggested that Vangl2 plays a crucial role in cartilage development and disease. An in vitro study revealed that Vangl2 participates in Wnt5a/PCP signaling that promotes the formation of organized columns of isolated growth plate chondrocytes [19]. Animal experiments also indicate that during skeletal development, Wnt5a-induced Vangl2 phosphorylation facilitates growth plate chondrocyte columnar organization and limb growth [20, 21]. In addition, mutated Vangl2 in mice results in shortened limbs and reduced bone volume [21-23]. However, despite these findings, our understanding of the precise role of Vangl2 in OA chondrocytes is limited. In this study, we demonstrate for the first time that there is a link between Vangl2 and
OA process and investigate the potential molecular mechanisms.

\section{Materials and methods Chondrocytes culture}

ATDC5 cells (Fuheng Centre Cell Bank, China), a murine chondrogenic cell line, were cultured in Dulbecco's modified Eagle's medium/Ham's F-12 nutrient mixture (DMEM/F-12; Gibco, USA) containing 10\% fetal bovine serum (FBS; Gibco) and $1 \%$ penicillin/streptomycin (Gibco, USA). Cells were maintained in a humidified atmosphere containing $5 \% \mathrm{CO} 2$ at $37{ }^{\circ} \mathrm{C}$. After the cells had reached $80-90 \%$ confluence, they were collected for experimentation. The medium was changed every 2 days. Then, ATDC5 were treated with recombinant mouse IL$1 \beta(10 \mathrm{ng} / \mathrm{mL})$ to simulate the inflammatory environment of OA.

\section{Cell transfection}

Chondrocytes were inoculated into 6-well plates (Corning, NY, USA) at a density of $5 \times 10^{5}$ cells/well (Corning, NY, USA). According to the manufacturer's instructions, the Vangl2 small interfering RNA (siRNA; RiboBio; Guangzhou, China) and negative control (nc; RiboBio; Guangzhou, China) were transfected by using Lipofectamine RNAiMAX (Invitrogen, CA, USA) when the cell fusion rate reached $50-70 \%$. To establish Wnt5a overexpressing cells, we used Lipofectamine 2000 (Invitrogen, CA, USA) to transfect plasmids targeting Wnt5a and negative control (NC) after cells reached $70 \%$ confluence. The medium was replaced with DMEM/F12 supplemented with $10 \%$ FBS after $6 \mathrm{~h}$.

\section{RNA quantification by real-time quantitative polymerase} chain reaction (RT-qPCR) amplification

Total cell RNA was extracted by using TRIzol. The mRNA was mixed with the PrimeScript RT Master Mix (Perfect Real Time; Takara, Japan) and RNase-free water, then reverse-transcribed into cDNA according to the manufacturer's instructions. The primers used for RTqPCR were listed in Table 1. qPCR was performed by the SYBR ${ }^{\otimes}$ Green RT-qPCR kit (Roche Diagnostics, Switzerland) and a Roche light cycle 96 (Roche, Switzerland). The GAPDH mRNA expression level is used for normalization, and the mRNA expression levels were calculated using the $2^{-\Delta \Delta \mathrm{cq}}$ method. We assigned an arbitrary value of 1 for each control expression level. The treated samples were evaluated as fold change over control.

\section{Western blotting}

Cells were treated by RIPA lysis buffer (Beyotime Institute of Biotechnology, China) supplemented with $1 \%$ protease and phosphatase inhibitors (Beyotime, China). 
Table 1 Primer sequences for PCR

\begin{tabular}{|c|c|c|}
\hline Gene (mouse) & Primer sequence $\left(3^{\prime} \rightarrow 5^{\prime}\right)$ forward & Primer sequence $\left(3^{\prime} \rightarrow 5^{\prime}\right)$ reverse \\
\hline Wnt5a & ATGCAGTACATTGGAGAAGGTG & CGTCTCTCGGCTGCCTATTT \\
\hline Vangl2 & GGGATGGGAGTCGTGGAGATA & TCATGGGAGATACTGTGCTCAG \\
\hline MMP3 & ACATGGAGACTTTGTCCCTITTG & TTGGCTGAGTGGTAGAGTCCC \\
\hline MMP9 & CTGGACAGCCAGACACTAAAG & CTCGCGGCAAGTCTTCAGAG \\
\hline MMP13 & CTATCCCTTGATGCCATTACCAG & ATCCACATGGTTGGGAAGTTC \\
\hline Col-2 & CAGGATGCCCGAAAATTAGGG & ACCACGATCACCTCTGGGT \\
\hline IL-6 & CCAAGAGGTGAGTGCTTCCC & CTGTTGTTCAGACTCTCTCCCT \\
\hline IL-8 & CAAGGCTGGTCCATGCTCC & TGCTATCACTTCCTITCTGTTGC \\
\hline TNF-a & GACGTGGAACTGGCAGAAGAG & TTGGTGGTTTGTGAGTGTGAG \\
\hline
\end{tabular}

Then, a bicinchoninic assay (Beyotime, China) was used to measure protein concentration. Protein samples were added to the loading buffer (loading buffer to sample ratio, 1:4) and were heated to $99^{\circ} \mathrm{C}$ for $10 \mathrm{~min}$ to denature the proteins. Eight percent or $10 \%$ sodium dodecyl sulfate-polyacrylamide gel electrophoresis (Beyotime, China) was used to separate proteins, followed by transferring to polyvinylidene fluoride membranes (Millipore, USA) and by blocking with $5 \%$ non-fat milk (BD Biosciences, USA) for $1 \mathrm{~h}$ at room temperature (RT). The blot was incubated with primary antibodies (1:1,000 dilutions) overnight at $4{ }^{\circ} \mathrm{C}$ (rabbit antibodies against MMP3, MMP9 , MMP13, IL-6, aggrecan, p38, phosphorylated (p)-p38, and GAPDH (Affinity Biosciences, USA); rabbit antibodies against ERK, p-ERK, JNK, pJNK, p65, p-p65 (Cell signaling Technology, USA), and Col-2 (1:1000; Bioss, China); and mouse antibodies against Vangl2 (1:400; Santa Cruz, USA)). Then, the blots were washed for 5 min 5 times by phosphatebuffered saline supplemented with $0.05 \%$ Tween 20

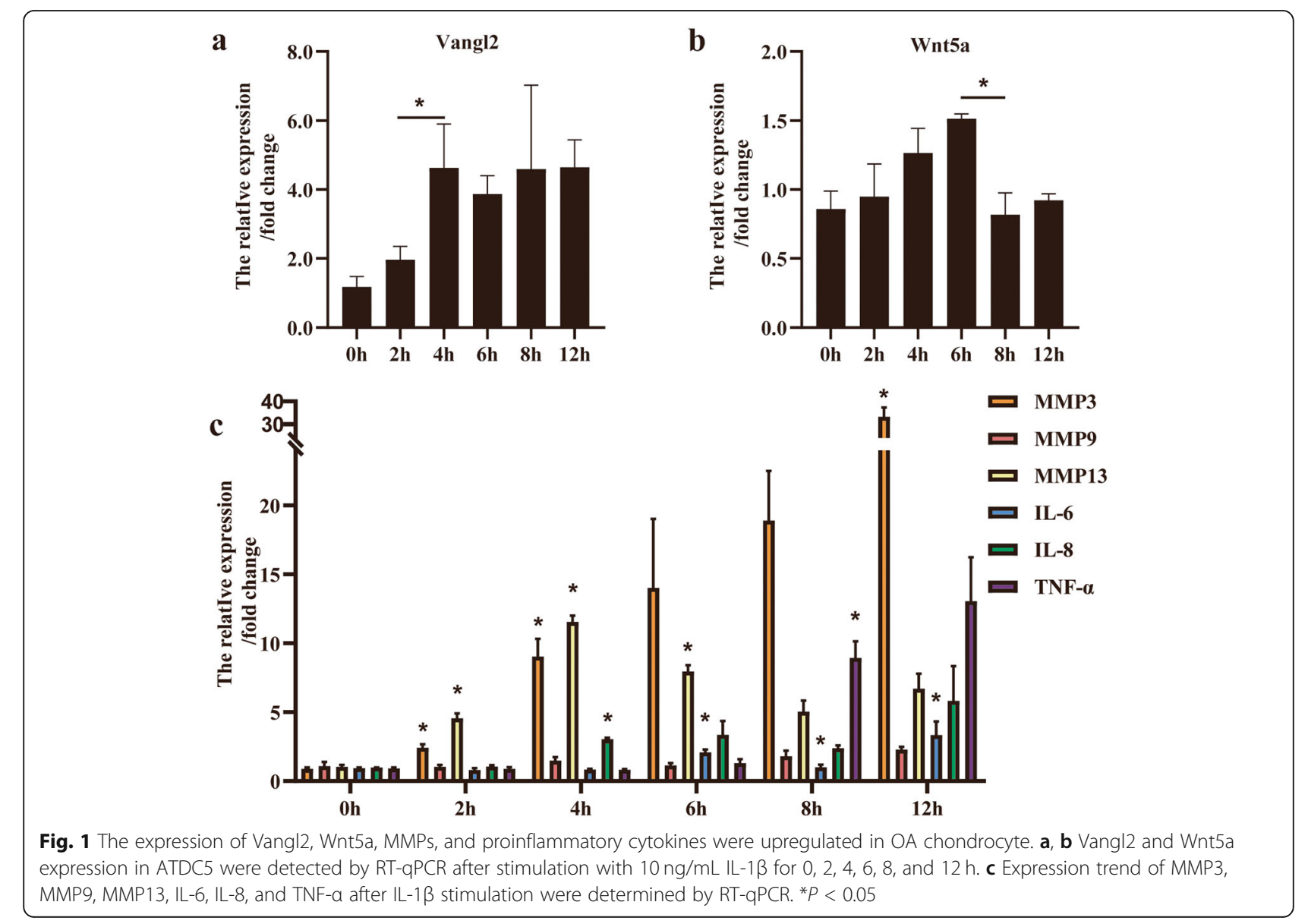




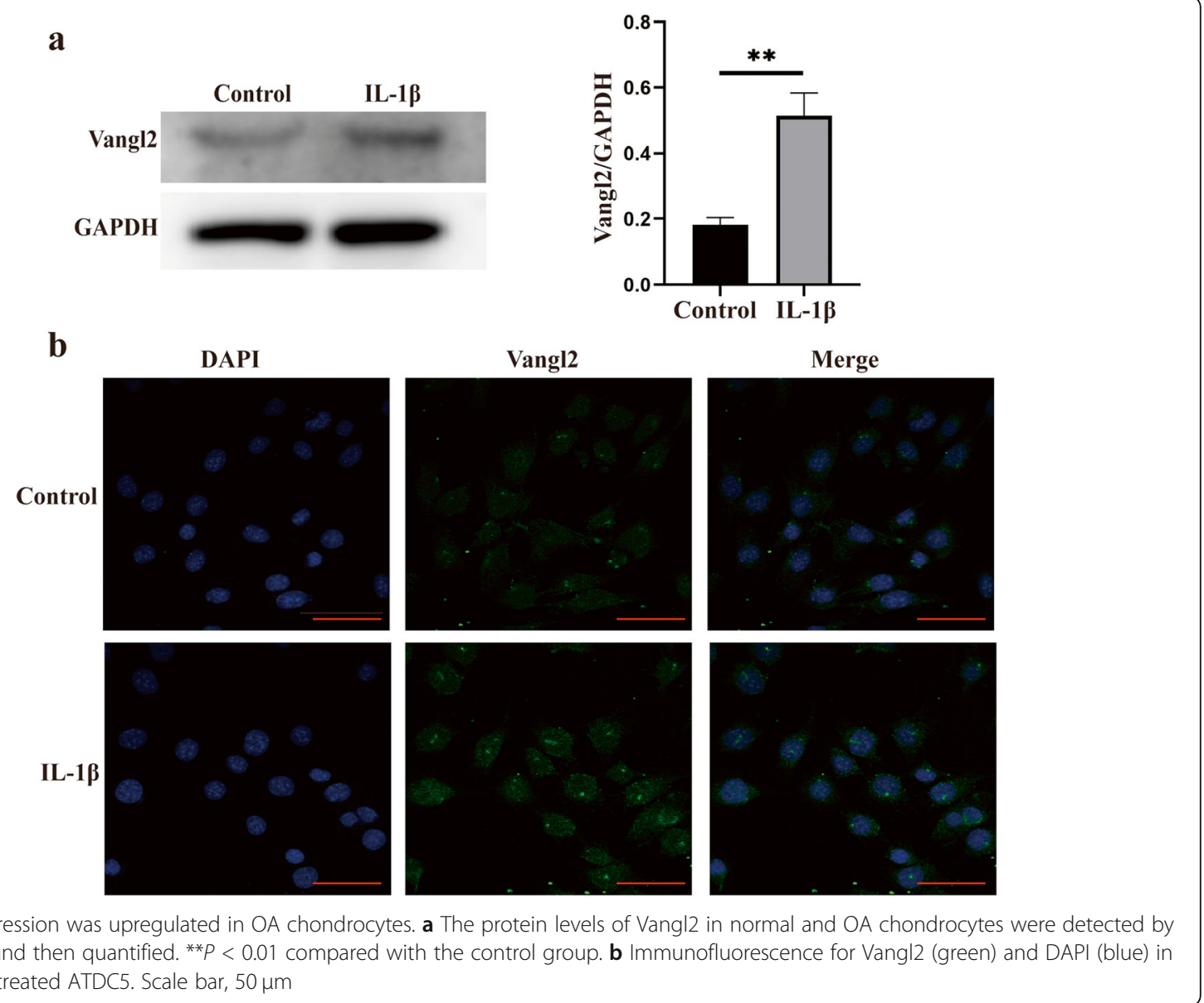

(TBST) at RT. Then, they were incubated with a horseradish peroxide-conjugated goat anti-rabbit or antimouse IgG (1:2000; Cell Signaling Technology, USA) for $1 \mathrm{~h}$ (RT). An ECL Kit (Millipore, USA) was used to visualize the protein blots and Image J (version: $1.52 \mathrm{t}$; National Institutes of Health) was used for semiquantitative analysis.

\section{Cell immunocytofluorescence (IF)}

5000 ATDC5 were seeded in a 35-mm dish for confocal laser-scanning microscopy (MatTek Corporation, USA). Cells were treated after $24 \mathrm{~h}$ and then the culture medium was discarded. After being washed with PBS three times, the cells were fixed with $4 \%$ paraformaldehyde (Beyotime, China) for $15 \mathrm{~min}$ (RT). Subsequently, the cells were permeabilized with $0.1 \%$ Triton X-100 and blocked in 5\% bovine serum albumin (BSA) for $1 \mathrm{~h}$. Mouse anti-Vangl2 (1:400, Santa Cruz, USA) antibody was added to the dishes for incubation at $4{ }^{\circ} \mathrm{C}$ overnight. Cells were incubated in the dark for $1 \mathrm{~h}$ with secondary antibodies (488 DyLight goat anti-mouse IgG; 1:100; EarthOx, USA). The nuclei were counterstained with 4',6-diamidino-2-phenylindole (DAPI; Beyotime, China) for $5 \mathrm{~min}$ after being washed with
PBS. Then, the cells were observed under a fluorescence microscope (Carl Zeiss, Germany).

\section{Immunocytochemical (ICC) staining}

The transfected ATDC5 were grown on glass coverslips that were previously coated with poly L-lysin. Then, they were fixed in 4\% paraformaldehyde (Beyotime, China) for 15 min (RT). Permealization was done by immersing slides in $0.3 \%$ Triton-X100 (MP Biomedicals, USA) for 15 min. Two drops of the rabbit primary antibody against Col-2 (1:400) were applied to each slide. Then, the samples were incubated at $4{ }^{\circ} \mathrm{C}$ overnight. The next day, the Horseradish peroxidase-conjugated secondary antibody (goat antirabbit) was applied to each slide for $1 \mathrm{~h}$. Subsequently, DAB (Zhongshan Jinqiao Biotechnology, China) was prepared and was used for visualizing any antigen-antibody reaction in the cells. The stained sections were observed and recorded by a light microscope (Leica, German).

\section{Statistical analysis}

All statistical calculations were performed using SPSS 25.0 (IBM, USA) and each assay was performed with at least three technical and biological replicates. Normally 


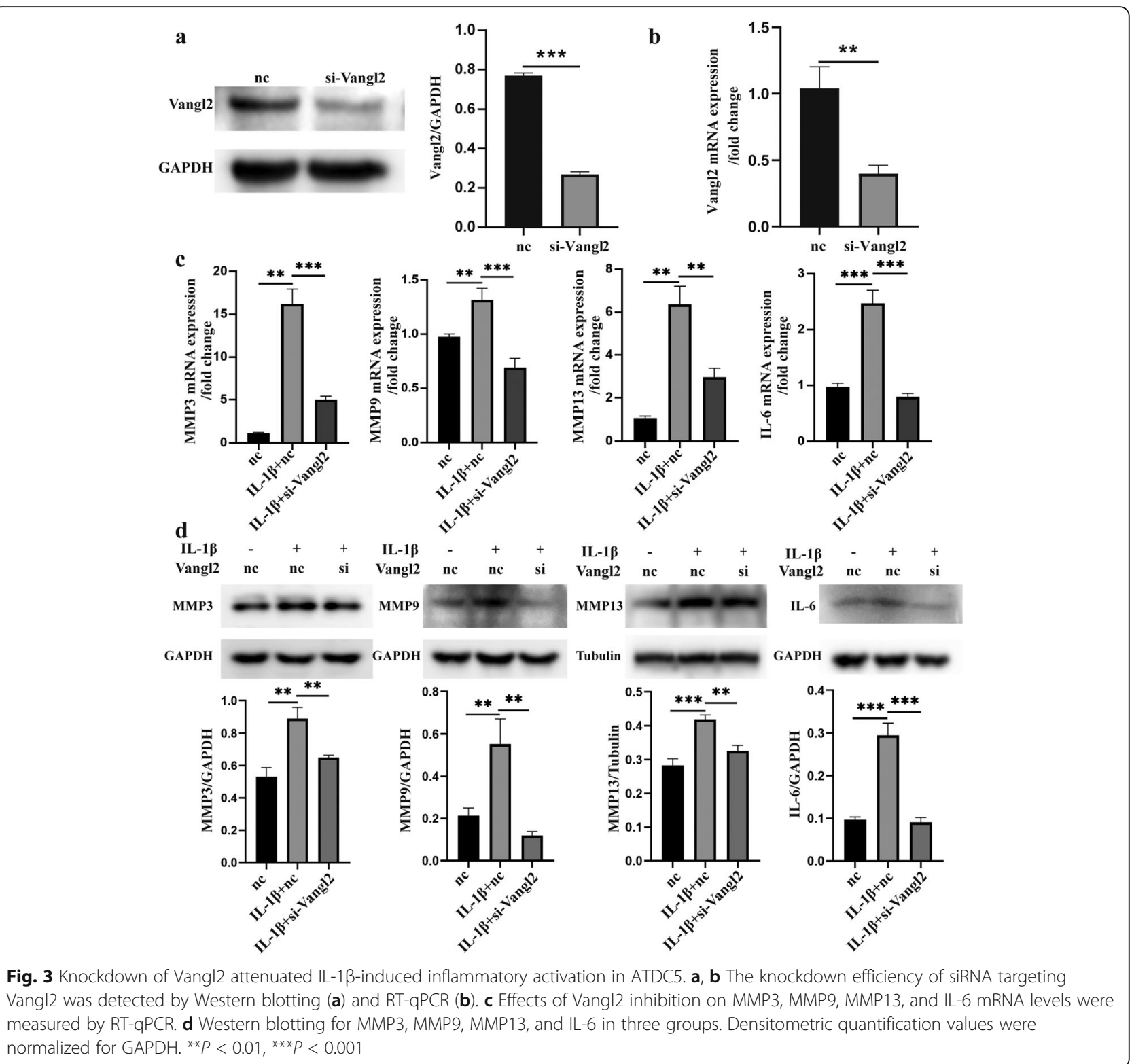

distributed data were expressed as means \pm standard deviations. Students $t$ test was used to analyze the differences between the two groups. Significant differences between more than two groups were determined by the analysis of variance (ANOVA). $P<0.05$ was considered to indicate a statistically significant difference.

\section{Results}

The expression of Vangl2, Wnt5a, and MMPs increased after IL-1 $\beta$ stimulation

Exogenous IL-1 $\beta(10 \mathrm{ng} / \mathrm{mL})$ enhanced the mRNA expression of Vangl2, Wnt5a, MMPs, and related proinflammatory factors in a time-dependent manner. The time-course study showed that the expression of
Vangl2, MMP3, and MMP13 increased at early stages. Vangl2 reached its peak at $4 \mathrm{~h}$ and remained at a high level for the rest of period (Fig. 1a). The expression of Wnt5a gradually elevated after IL- $1 \beta$ treatment and then decreased after $6 \mathrm{~h}$ (Fig. 1b). Although differences of MMP9 expression between adjacent time points were not statistically significant, all mRNA levels of MMP3, MMP9, MMP13, IL-6, IL-8, and TNF- $\alpha$ increased in IL-1 $\beta$-treated chondrocyte (Fig. $1 c)$. The expression of MMP3 changed most significantly after IL-1 $\beta$ stimulation and almost reached at 35 times of the initial level at $12 \mathrm{~h}$. MMP13 expression also increased around 12 times of the initial value at $4 \mathrm{~h}$. 


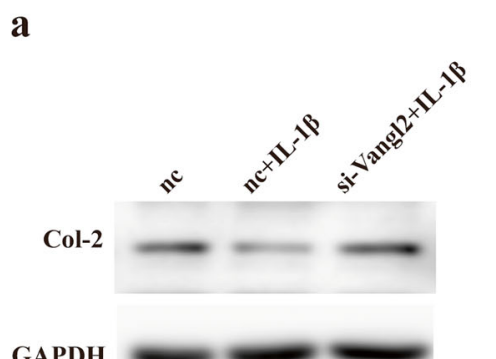

GAPDH
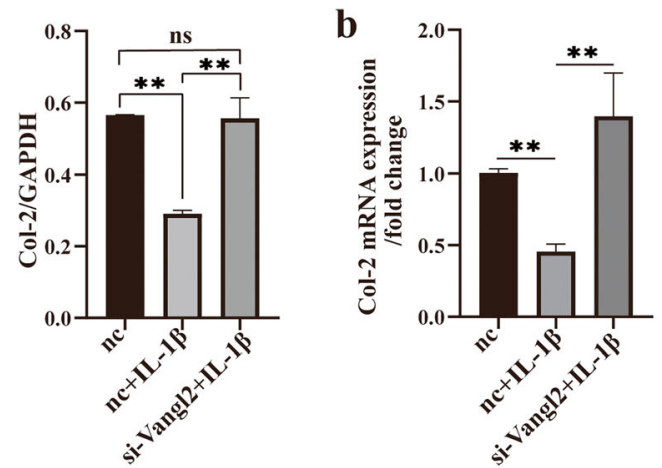

c
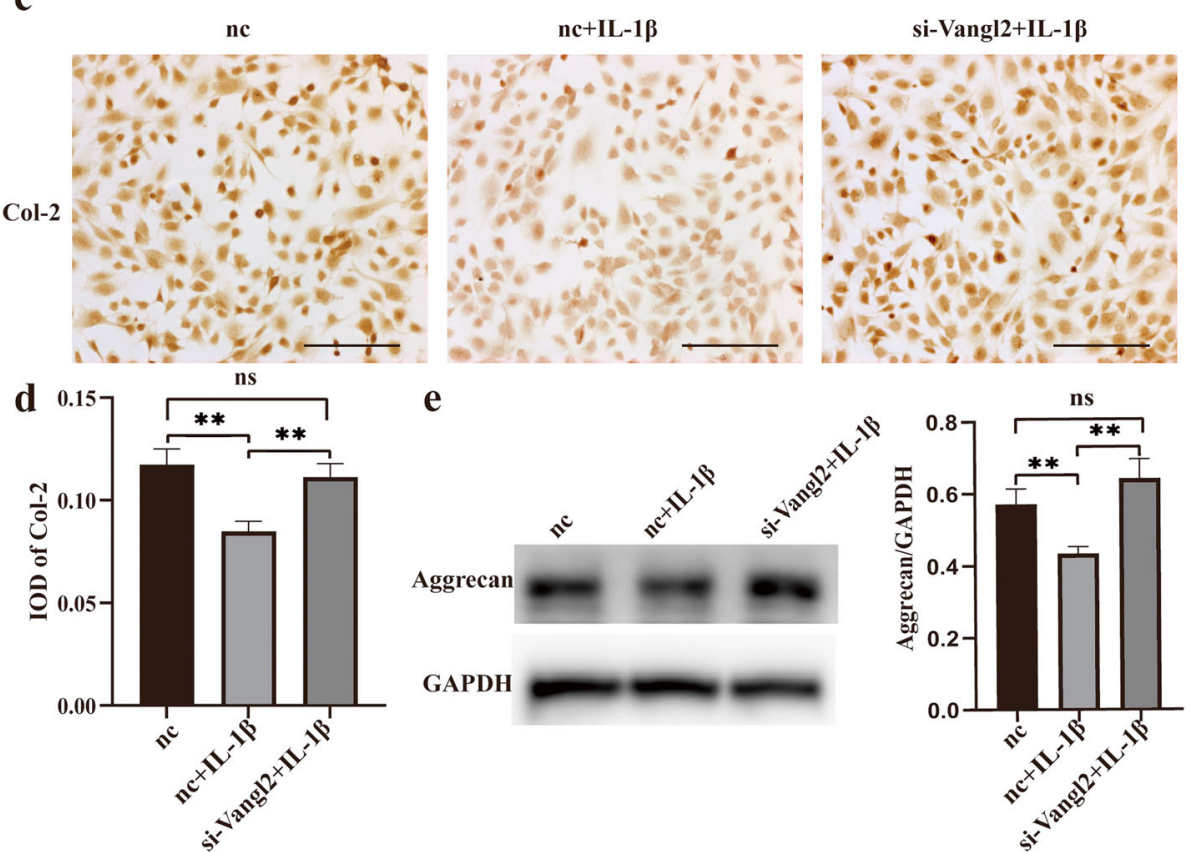

Fig. 4 Silencing of Vangl2 prevents Col-2 and aggrecan degradation in OA. a Western blotting of Col-2 in three groups. The images were quantitatively analyzed and normalized to GAPDH. $\mathbf{b}$ The change of Col-2 mRNA was quantitated by RT-qPCR. Representative ICC images of Col-2 (c) and the IOD analysis (d). Scale bar, $50 \mu \mathrm{m}$. e The aggrecan expression was measured by Western blotting and quantified for three groups. ns, not statistically significant, ${ }^{*} P<0.05,{ }^{*} P<0.01 ; \mathrm{IOD}$, integrated option density

Induction of Vangl2 protein by IL-1 $\beta$ was further verified in ATDC5 by Western blotting and IF analysis (Fig. 2a, b). A significant increase of Vangl2 immunostaining was observed after stimulation with IL$1 \beta$ for $4 \mathrm{~h}$, showing the consistent effects of both the mRNA and protein levels. Moreover, the increased level of Vangl2 in OA chondrocytes is also confirmed by IF staining (Fig. 2b).

Knockdown of Vangl2 alleviated IL-1 $\beta$-induced MMP 3, MMP9, MMP13, and IL-6 expression at both the gene and protein levels

To further evaluate the effects of Vangl2 on the inflammatory response of OA chondrocyte, we analyzed whether suppression of endogenous Vangl2 modulates the expression of MMPs and IL-6. Western blotting and RT-qPCR showed that the knockdown efficiency of siRNA targeting Vangl2 was around 50\% (Fig. 3a, b). The chondrocytes were divided into three groups: group I (nc), chondrocytes were only transfected by siRNA of nc; group II $(\mathrm{IL}-1 \beta+\mathrm{nc})$, cells were further stimulated with IL-1 $\beta$; and group III (IL-1 $\beta+$ si-Vangl2), OA chondrocytes were transfected with siRNA targeting Vangl2. The RT-qPCR and Western blotting analysis showed that gene expressions of MMP3, MMP9, MMP13, and IL-6 were significantly increased in IL- $1 \beta$ stimulation compared with the nc group (Fig. 3c, d). Then, we found that Vangl2 silencing notably alleviated the IL-1 $\beta$ induced gene expression of MMPs and IL-6 (Fig. 3c). Western blotting also revealed that knockdown of 
Vangl2 reduced the protein expression of MMP3, MMP9, MMP13, and IL-6 significantly (Fig. 3d).

Vangl2 silencing reduces the loss of cartilage ECM in vitro To explore the relationship between Vangl2 and chondrocyte anabolism in OA, we analyzed the expression of the major structural proteins in cartilage ECM, Col-2, and aggrecan (Fig. 4). As shown in the Fig. 4, the protein and gene levels of Col-2 declined to approximately $50 \%$ after stimulated with IL-1 $\beta$ (Fig. $4 a, b)$. It is of note that Vangl2 inhibition almost eliminates the effects of IL-1 $\beta$ on Col-2 and aggrecan. Both Western blotting and RT-qPCR showed that the expression levels of Col-2 in group III returned to normal (Fig. 4a, b). The results of ICC staining further confirmed our findings (Fig. 4c, d). Similarly, Vangl2 silencing also notably suppressed the degradation of aggrecan in OA chondrocytes (Fig. 4e).

\section{Inhibition of Vangl2 attenuates the proinflammatory} functions of Wnt5a and the underlying mechanism We constructed Wnt5a-overexpressing chondrocytes by plasmid transfection and investigated the role of
Wnt5a in the IL-1 $\beta$-induced inflammatory response of chondrocytes (Fig. 5a). Wnt5a promoted the reduction of Sox9, Col-2, and the upregulation of MMP3, MMP9, MMP13 in OA (Fig. 5b, c). As expected, Vangl2 silencing almost eliminated the proinflammatory effects of Wnt5a in OA chondrocyte (Fig. 5b, c). Specifically, the expression of Sox9 and Col-2 was upregulated and the expression of MMP3, MMP9, and MMP13 was reduced in Vangl2silencing chondrocyte.

To further investigate the mechanisms by which Vangl2 silencing protects chondrocytes, we examined the phosphorylation levels of P38 MAPK, extracellular signal-regulated kinase (ERK), c-Jun NH2-terminal kinase (JNK), and P65 by Western blotting (Fig. 6). The results showed that Wnt5a further upregulated the expression of phosphorylated-P38, JNK, ERK, and P65, which had been induced by IL-1 $\beta$ (Fig. 6a, c). Knockdown of Vangl2 significantly inhibited the expression of P-P38, P-JNK, and P-ERK induced by Wnt5a (Fig. 6a, b). Simultaneously, Vangl2 silencing also notably decreased the phosphorylation level of P65 enhanced by Wnt5a (Fig. 6c).

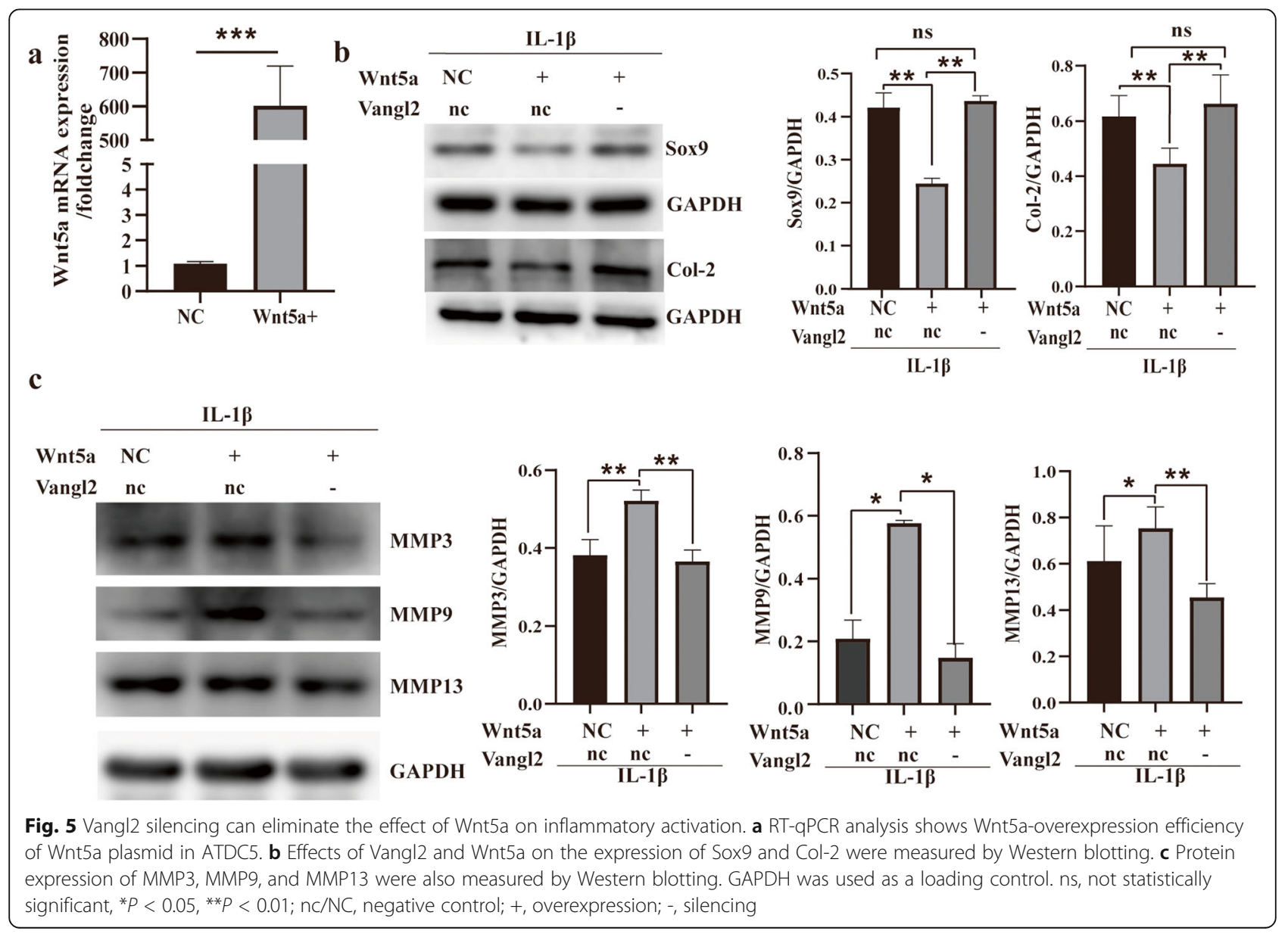




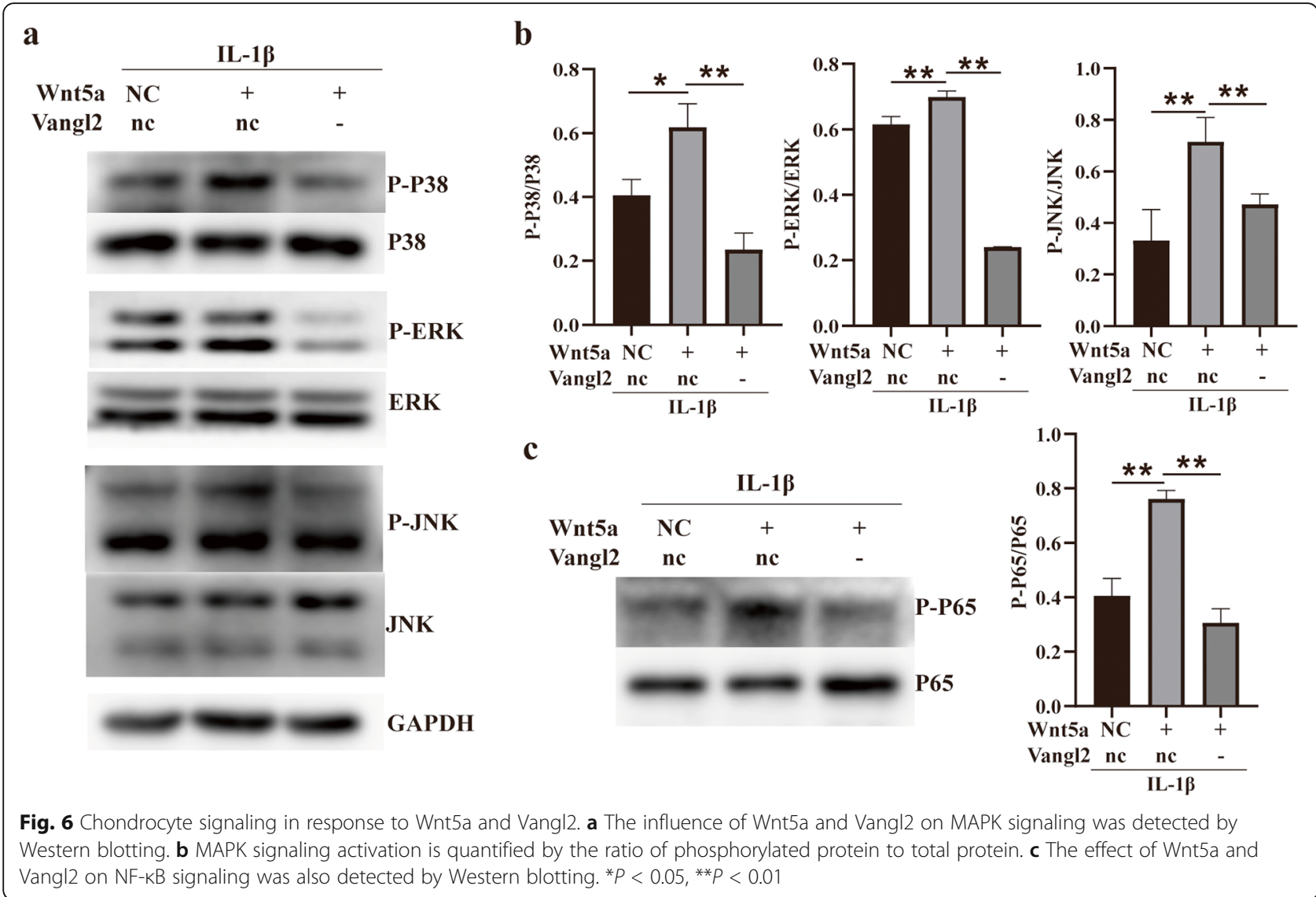

\section{Discussion}

Cellular functions of Vangl2 have been explored for more than two decades since Vangl2/Stbm was first identified in Drosophila [24]. A previous study in human revealed that the expression of Vangl2 increased in the OA synovium [17]. Thus, our finding that Vangl2 was significantly increased in $\mathrm{OA}$ chondrocytes raised the question of whether Vangl2 plays a role in OA progression. In this study, we demonstrate for the first time that Vangl2 is involved in OA progression, and Vangl2 silencing can suppress the proinflammatory functions of Wnt5a in OA chondrocytes.

In our study, the upregulation of Vangl2 was determined at an early stage during IL- $1 \beta$ stimulation, which is similar with MMPs. This finding suggests a link between Vangl2 and MMPs, the key mediators of the cartilage destruction in OA [25]. Our further experiments provide evidence that knockdown of Vangl2 corrected the imbalance between anabolic and catabolic factors in IL-1 $\beta$-treated chondrocytes, resulting in the inhibition of MMP3, MMP9, MMP13, and IL-6 as well as the upregulation of Col-2 and aggrecan. In this study, MMP3 and MMP13 were significantly suppressed by Vangl2 silencing. Surprisingly, we found that the main gelatinases, MMP9, decreased to the level of control group in
Vangl2-silencing chondrocyte. These MMPs are major contributors to the degenerative process in OA due to their digestion of cartilage ECM $[25,26]$. Among them, MMP13 can cleave the triple helix structure of Col-2, the predominant collagen in cartilage, and other MMPs can further digest the collagen hydrolysate, as well as aggrecan [27]. These results confirmed our notion that Vangl2 regulates MMPs in OA chondrocytes, which is also supported by prior findings that Vangl2 can directly regulate MMPs in other cells [28, 29]. Moreover, Vangl2 silencing restored the expression of Col-2 and aggrecan, which are the main structural proteins of cartilage ECM. Collectively, our results indicate that Vangl2 silencing protects chondrocyte from IL- $1 \beta$-induced metabolic disorder in vitro.

Unexpectedly, we found that Vangl2 silencing almost eliminates the proinflammatory effects of Wnt5a in OA chondrocytes in this experiment. The increased expression of MMP3, MMP9, and MMP13 by Wnt5a was significantly suppressed by the inhibition of Vangl2. Notably, the expression of Sox9, a key transcription factor related to cartilage formation, and Col-2, returned to the baseline levels after Vangl2 silencing. Our finding that Wnt5a promotes inflammation via multiple pathways in OA chondrocytes is consistent with the results 
reported before $[9,30,31]$. These data show that all three MAPK pathways (p38, ERK, JNK) can be activated by Wnt5a in OA chondrocytes. The MAPK pathway is one of the most important signal transduction systems in OA pathogenesis, which regulates cartilage ECM degradation (MMPs production) and synthesis (Sox9/Col-2 expression) [32-34]. Furthermore, we observed a marked upregulation of p-p65 (the representative protein of NF- $\mathrm{kB}$ pathway) in Wnt5a-overexpressing chondrocytes. NF- $\mathrm{KB}$ is an essential transcription factor that, together with other pathways including the MAPK pathway, mediates inflammation and regulates catabolism and apoptosis of chondrocytes in OA [35-37]. More importantly, we found that Vangl2 knockdown remarkably decreased the activation of MAPK and NF- $\mathrm{kB}$ pathway that induced by Wnt5a in this study. In general, our results reveal that Vangl2 silencing notably suppresses the proinflammatory functions of Wnt5a via the MAPK and $\mathrm{NF}-\mathrm{kB}$ pathway in OA chondrocytes. Increasing our knowledge of Vangl2 may provide new opportunities for the development of OA treatment.

\section{Conclusion}

For the first time, we demonstrate that Vangl2 is involved in the OA process. Vangl2 silencing can notably alleviate OA progression in vitro by correcting the imbalance between anabolic and catabolic factors and can inhibit the proinflammatory effects of Wnt5a via MAPK and NF- $\mathrm{KB}$ pathway in IL-1 $\beta$-treated chondrocytes. This study provides new insight into the mechanisms of cartilage inflammation.

\section{Acknowledgements}

Not applicable

\section{Authors' contributions}

$Z \mathrm{ZH}$ and ZK conceived the study design. ZK, LZY, and LYY performed the study, collected the data, and contributed to the study design. ZK prepared the manuscript. $Z \mathrm{H}, \mathrm{XLY}$, and SJD edited the manuscript. All authors read and approved the final manuscript.

\section{Funding}

This research was financially supported by the National Natural Science Foundation of China (No. 81400491) and the Science and Technology Planning Project of Guangdong Province (2017A020215040).

\section{Availability of data and materials}

We state that the data will not be shared since all the raw data are present in the figures included in the article.

\section{Ethics approval and consent to participate}

Not applicable.

\section{Consent for publication}

Not applicable.

\section{Competing interests}

The authors declare that they have no competing interests.

\section{Author details}

${ }^{1}$ Hospital of Stomatology, Sun Yat-sen University, Guangdong Provincial Key Laboratory of Stomatology, Guanghua School of Stomatology, Sun Yat-sen University, No.56 Lingyuan West Road, Guangzhou 510055 Guangdong, People's Republic of China. ${ }^{2}$ Department of Stomatology, Binhaiwan Central Hospital of Dongguan (also called The Fifth People's Hospital of Dongguan), The Dongguan Affiliated Hospital of Medical College of Jinan University, No.111 Humen Road, Humen Town, Dongguan City 523905, Guangdong Province, People's Republic of China.

Received: 2 December 2020 Accepted: 28 January 2021

Published online: 15 February 2021

\section{References}

1. Loeser RF, Goldring SR, Scanzello CR, Goldring MB. Osteoarthritis: a disease of the joint as an organ. Arthritis Rheum. 2012;64(6):1697-707.

2. Martel-Pelletier J, Barr AJ, Cicuttini FM, Conaghan PG, Cooper C, Goldring MB, Goldring SR, Jones G, Teichtahl AJ, Pelletier JP. Osteoarthritis. Nat Rev Dis Primers. 2016;2:16072.

3. Berenbaum F, Wallace IJ, Lieberman DE, Felson DT. Modern-day environmental factors in the pathogenesis of osteoarthritis. Nat Rev Rheumatol. 2018;14(11):674-81.

4. Mobasheri A, Rayman MP, Gualillo O, Sellam J, van der Kraan P, Fearon U. The role of metabolism in the pathogenesis of osteoarthritis. Nat Rev Rheumatol. 2017;13(5):302-11.

5. Kapoor M, Martel-Pelletier J, Lajeunesse D, Pelletier JP, Fahmi H. Role of proinflammatory cytokines in the pathophysiology of osteoarthritis. Nat Rev Rheumatol. 2011:7(1):33-42.

6. Goldring MB, Otero M. Inflammation in osteoarthritis. Curr Opin Rheumatol. 2011;23(5):471-8.

7. Le Clanche S, Bonnefont-Rousselot D, Sari-Ali E, Rannou F, Borderie D. Interrelations between osteoarthritis and metabolic syndrome: a common link? Biochimie. 2016;121:238-52.

8. De Santis M, Di Matteo B, Chisari E, Cincinelli G, Angele P, Lattermann C, Filardo G, Vitale ND, Selmi C, Kon E. The role of Wnt pathway in the pathogenesis of $O A$ and its potential therapeutic implications in the field of regenerative medicine. Biomed Res Int. 2018;2018:7402947.

9. Huang G, Chubinskaya S, Liao W, Loeser RF. Wnt5a induces catabolic signaling and matrix metalloproteinase production in human articular chondrocytes. Osteoarthritis Cartilage. 2017;25(9):1505-15.

10. Martineau X, Abed É, Martel-Pelletier J, Pelletier JP, Lajeunesse D. Alteration of Wnt5a expression and of the non-canonical Wnt/PCP and Wnt/PKC-Ca2+ pathways in human osteoarthritis osteoblasts. PLoS One. 2017;12(8): e0180711.

11. Shi S, Man Z, Li W, Sun S, Zhang W. Silencing of Wnt5a prevents interleukin$1 \beta$-induced collagen type II degradation in rat chondrocytes. Exp Ther Med. 2016;12(5):3161-6.

12. Katoh $Y$, Katoh M. Comparative genomics on Vangl1 and Vangl2 genes. Int J Oncol. 2005;26(5):1435-40.

13. Hayes MN, McCarthy K, Jin A, Oliveira ML, lyer S, Garcia SP, Sindiri S, Gryder B, Motala Z, Nielsen GP, et al. Vangl2/RhoA signaling pathway regulates stem cell self-renewal programs and growth in rhabdomyosarcoma. Cell Stem Cell. 2018;22(3):414-27 e416.

14. Gao B, Song H, Bishop K, Elliot G, Garrett L, English MA, Andre P, Robinson J, Sood R, Minami Y, et al. Wnt signaling gradients establish planar cell polarity by inducing Vangl2 phosphorylation through Ror2. Dev Cell. 2011; 20(2):163-76.

15. Zhang K, Yao E, Lin C, Chou YT, Wong J, Li J, Wolters PJ, Chuang PT. A mammalian Wnt5a-Ror2-Vangl2 axis controls the cytoskeleton and confers cellular properties required for alveologenesis. Elife. 2020;9:e53688.

16. Hosseini-Farahabadi S, Geetha-Loganathan P, Fu K, Nimmagadda S, Yang HJ Richman JM. Dual functions for WNT5A during cartilage development and in disease. Matrix Biol. 2013:32(5):252-64.

17. Huang J, Chen C, Liang C, Luo P, Xia G, Zhang L, Wang X, Wen Z, Cao X, Wu S. Dysregulation of the Wnt signaling pathway and synovial stem cell dysfunction in osteoarthritis development. Stem Cells Dev. 2020;29(7):40113.

18. Tong W, Zeng Y, Chow DHK, Yeung W, Xu J, Deng Y, Chen S, Zhao H, Zhang $X$, Ho KK, et al. Wnt16 attenuates osteoarthritis progression through a PCP/JNK-mTORC1-PTHrP cascade. Ann Rheum Dis. 2019;78(4):551-61. 
19. Randall RM, Shao YY, Wang L, Ballock RT. Activation of Wnt planar cell polarity (PCP) signaling promotes growth plate column formation in vitro. J Orthop Res. 2012;30(12):1906-14.

20. Kato K, Bhattaram P, Penzo-Méndez A, Gadi A, Lefebvre V. SOXC Transcription factors induce cartilage growth plate formation in mouse embryos by promoting noncanonical WNT signaling. J Bone Miner Res. 2015;30(9):1560-71.

21. Yang W, Garrett L, Feng D, Elliott G, Liu X, Wang N, Wong YM, Choi NT, Yang Y, Gao B. Wnt-induced Vangl2 phosphorylation is dose-dependently required for planar cell polarity in mammalian development. Cell Res. 2017; 27(12):1466-84.

22. Wang B, Sinha T, Jiao K, Serra R, Wang J. Disruption of PCP signaling causes limb morphogenesis and skeletal defects and may underlie Robinow syndrome and brachydactyly type B. Hum Mol Genet. 2011;20(2):271-85.

23. Orriss IR, Lanham S, Savery D, Greene NDE, Stanier P, Oreffo R, Copp AJ, Galea GL. Spina bifida-predisposing heterozygous mutations in planar cell polarity genes and Zic2 reduce bone mass in young mice. Sci Rep. 2018; 8(1):3325

24. Wolff T, Rubin GM. Strabismus, a novel gene that regulates tissue polarity and cell fate decisions in Drosophila. Development. 1998;125(6):1149-59.

25. Mehana EE, Khafaga AF, El-Blehi SS. The role of matrix metalloproteinases in osteoarthritis pathogenesis: An updated review. Life Sci. 2019;234:116786.

26. Shi L, Liang T, Yang F, Zhu FF, Liu J, Jiang JQ, Wu XW, Chen AS, Yuan DP, Liang XL. Matrix metalloproteinase-3 induces proteoglycan degradation in gouty arthritis model. Gene. 2021;765:145120.

27. Andia I, Sanchez M, Maffulli N. Joint pathology and platelet-rich plasma therapies. Expert Opin Biol Ther. 2012;12(1):7-22.

28. Cantrell VA, Jessen JR. The planar cell polarity protein Van Gogh-Like 2 regulates tumor cell migration and matrix metalloproteinase-dependent invasion. Cancer Lett. 2010;287(1):54-61.

29. Papakrivopoulou E, Vasilopoulou E, Lindenmeyer MT, Pacheco S, Brzóska H, Price KL, Kolatsi-Joannou M, White KE, Henderson DJ, Dean CH, et al. Vangl2, a planar cell polarity molecule, is implicated in irreversible and reversible kidney glomerular injury. J Pathol. 2018;246(4):485-96.

30. Ge XP, Gan YH, Zhang CG, Zhou CY, Ma KT, Meng JH, Ma XC. Requirement of the NF-KB pathway for induction of Wnt-5A by interleukin-1 $\beta$ in condylar chondrocytes of the temporomandibular joint: functional crosstalk between the Wnt-5A and NF-KB signaling pathways. Osteoarthritis Cartilage. 2011; 19(1):111-7.

31. Rodriguez-Trillo A, Mosquera N, Pena C, Rivas-Tobío F, Mera-Varela A, Gonzalez A, Conde C. Non-canonical WNT5A signaling through RYK contributes to aggressive phenotype of the rheumatoid fibroblast-like synoviocytes. Front Immunol. 2020;11:555245.

32. Zhang Y, Pizzute T, Pei M. A review of crosstalk between MAPK and Wnt signals and its impact on cartilage regeneration. Cell Tissue Res. 2014;358(3): 633-49.

33. Han Z, Boyle DL, Chang L, Bennett B, Karin M, Yang L, Manning AM, Firestein GS. c-Jun N-terminal kinase is required for metalloproteinase expression and joint destruction in inflammatory arthritis. J Clin Invest. 2001; 108(1):73-81.

34. Sondergaard BC, Schultz N, Madsen SH, Bay-Jensen AC, Kassem M, Karsdal MA. MAPKs are essential upstream signaling pathways in proteolytic cartilage degradation--divergence in pathways leading to aggrecanase and MMP-mediated articular cartilage degradation. Osteoarthritis Cartilage. 2010; 18(3):279-88.

35. Choi MC, Jo J, Park J, Kang HK, Park Y. NF-KB signaling pathways in osteoarthritic cartilage destruction. Cells. 2019;8(7):734.

36. Rigoglou S, Papavassiliou AG. The NF-KB signalling pathway in osteoarthritis. Int J Biochem Cell Biol. 2013;45(11):2580-4.

37. Andia I, Maffulli N. Platelet-rich plasma for managing pain and inflammation in osteoarthritis. Nat Rev Rheumatol. 2013;9(12):721-30.

\section{Publisher's Note}

Springer Nature remains neutral with regard to jurisdictional claims in published maps and institutional affiliations.

\section{Ready to submit your research? Choose BMC and benefit from:}

- fast, convenient online submission

- thorough peer review by experienced researchers in your field

- rapid publication on acceptance

- support for research data, including large and complex data types

- gold Open Access which fosters wider collaboration and increased citations

- maximum visibility for your research: over $100 \mathrm{M}$ website views per year

At BMC, research is always in progress.

Learn more biomedcentral.com/submissions 